\title{
Small polydispersed circular DNA contains strains of mobile genetic elements and occurs more frequently in permanent cell lines of malignant tumors than in normal lymphocytes
}

\author{
HANNELORE SCHMIDT ${ }^{1}$, HELGE TAUBERT ${ }^{1,3}$, HEIDEMARIE LANGE $^{1}$, KAREN KRIESE $^{1}$, \\ WOLFGANG DANIEL SCHMITT ${ }^{1}$, STEVE HOFFMANN ${ }^{2}$, FRANK BARTEL ${ }^{1}$ and STEFFEN HAUPTMANN ${ }^{1}$ \\ ${ }^{1}$ Institute of Pathology, Martin Luther University Halle Wittenberg, Halle; \\ ${ }^{2}$ Department of Computer Science, University of Leipzig, Leipzig, Germany
}

Received March 24, 2009; Accepted May 14, 2009

DOI: $10.3892 /$ or_00000450

\begin{abstract}
Small polydispersed circular DNA (spcDNA) belongs to the extrachromosomal pool of DNA and is composed of heterogeneous DNA circles. Whether spcDNA has a special function is currently unclear but their occurrence was suggested to be linked to genetic instability. In this study we investigated as to whether human lymphocytes from healthy volunteers also harbour spcDNA and whether spcDNA is present in all permanent cell lines from human normal and malignant tissues. Moreover, we were interested to see whether spcDNA contains sequences of mobile genetic elements. Our results show that spcDNA is present in all samples investigated yet the amount is lower in normal lymphocytes when compared to cancer cell lines (5.4 vs. 17.8\%). Alu sequences were present in 12/16 cancer cell lines whereas LINE-1 (L1) sequences were present in 15 of them. Six tumor cell lines also contained telomeric sequences. In contrast to that, spcDNA of normal lymphocytes contains Alu and L1 sequences only in $3 / 16$ cases and no telomeric sequences at all. Our findings suggest a direct dependency of the amount of Alu and L1 sequences on that of spcDNA. Beside these repetitive sequences, sequencing of spcDNA revealed in
\end{abstract}

Correspondence to: Dr Hannelore Schmidt, Institute of Pathology, University of Halle-Wittenberg, Magdeburger Strasse 14, D-06097 Halle/Saale, Germany

E-mail: hannelore.schmidt@medizin.uni-halle.de

Present address: ${ }^{3}$ Department of Oral and Maxillofacial Plastic Surgery, Martin Luther University Halle Wittenberg, Halle, Germany

Abbreviations: spcDNA, small polydispersed circular DNA; L1, LINE-1; tel-spcDNA, telomeric small polydispersed circular DNA; 2D, two-dimensional; LMW DNA, low-molecular-weight DNA; EtBr, ethidium bromide

Key words: spcDNA, Alu, LINE-1, telomeric sequences most cases chromosomal sequences of almost all chromosomes without an increased frequency of single regions. We suggest that the whole spcDNA including retrotranspositional elements and telomeric sequences may play a role for chromosomal rearrangements and genomic instability.

\section{Introduction}

The genome of the eukaryotes is comprised of chromosomal DNA and extrachromosomal elements which are DNA structures physically separated from the chromosomes. Small polydispersed circular DNA (spcDNA) as one such group of extrachromosomal elements is composed of heterogeneous circles that range in size from hundreds to several tens of kilobase pairs (1). The occurrence of spcDNA has been described both in normal physiological courses like ageing and in pathological processes associated with genomic instability (1-9). Various mechanisms such as chromosomal recombination and rearrangements as well as replication or transposition events were suggested to be involved in the generation and propagation of spcDNA (1). Indeed, such DNA segments can be excised from the genome by intrachromosomal homologous recombination between members of a repetitive sequence family. Cohen et al described a DNA ligase IV-dependent pathway of satellite DNA circle formation in the mouse genome (8).

We postulated the hypothesis that in the course of tumor development, it comes to an activation of mobile genetic elements, particularly of the LINE-1 (L1) family, and we wondered if this process might be accompanied by the occurrence of spcDNA. Moreover, we were interested to learn as to whether spcDNA can be regarded as a pool of fragmented extrachromosomal L1 elements.

In the spcDNA of some human and rodent cell lines and cancers there were double-stranded circular DNA molecules detected that apparently contained only telomeric repeats (tel-spcDNA) (4). However, to date there is no study that investigated the occurrence of tel-spc in normal human lymphocytes of healthy probands in comparison to cancer cell lines.

With the help of neutral-neutral two-dimensional (2D) gel electrophoresis we have analyzed a series of human cell lines 
Table I. Cell lines.

\begin{tabular}{|c|c|c|c|c|}
\hline Cell lines name & Catalog no. & Description & Reference & Medium \\
\hline HOSE & & Ovarian surface epithelium & Tsao et al, (11) & M199/MCDB105 (1/1) \\
\hline CAOV-3 & НТВ 75 & Ovarian adenocarcinoma & ATCC & DMEM \\
\hline ES-2 & CRL-1978 & Ovarian adenocarcinoma & ATCC & DMEM \\
\hline OAW-42 & & Ovarian adenocarcinoma & DKFZ, Heidelberg & DMEM \\
\hline OVCAR-3 & HTB 161 & Ovarian adenocarcinoma & ATCC & DMEM \\
\hline SK-OV-3 & HТB 77 & Ovarian adenocarcinoma & ATCC & DMEM \\
\hline MCF-10a & CRL-10317 & Breast epithelium (ductal) & ATCC & MEBM \\
\hline MCF-7 & НTB 22 & Breast adenocarcinoma & ATCC & DMEM \\
\hline $\mathrm{CX}-2$ & & Colon adenocarcinoma & DKFZ, Heidelberg & RPMI \\
\hline HRT-18 & CCL-244 & Colon adenocarcinoma & ATCC & RPMI \\
\hline HT-29 & HTB 38 & Colon adenocarcinoma & ATCC & RPMI \\
\hline HepG-2 & HB 8065 & Hepatocellular carcinoma & ATCC & DMEM \\
\hline Huh-7 & & Hepatocellular carcinoma & University, Hannover & DMEM \\
\hline SK-MEL-1 & НТВ 67 & Malignant melanoma & ATCC & DMEM \\
\hline U-2 OS & НТВ 96 & Osteogenic sarcoma & ATCC & DMEM \\
\hline SAOS-2 & HTB-85 & Osteogenic sarcoma & ATCC & RPMI \\
\hline SK-LMS-1 & НTB-88 & Leiomyosarcoma & ATCC & RMPI \\
\hline US 8-93 & & Undifferentiated sarcoma & Pathology, Halle & RPMI \\
\hline
\end{tabular}

ATCC, American Type Culture Collection.

of various origin (normal tissues and malignant tumors) as well as lymphocytes of healthy probands for the presence of spcDNA. Cloning and Southern hybridization were used to investigate as to whether spcDNA contains L1 and Alu elements.

\section{Materials and methods}

Normal lymphocytes and cell lines. Lymphocytic DNA of 16 healthy probands as well as DNA of 18 cell lines of various origins were studied for the occurrence of spcDNA. The investigated normal cell lines, MCF-10a, established from mammary epithelial cells (10) and HOSE, derived from ovarian epithelium (11), are both immortalized cell lines that were nontumorigenic in nude mice.

DNA was extracted using standard protocols (12). Descriptions, culture media and references of examined cell lines are listed in Table I. The cell lines were grown as monolayer supplemented with $10 \%$ fetal calf serum (Biowhittaker, Heidelberg, Germany) in a $37^{\circ} \mathrm{C}$ humidified atmosphere with $3 \% \mathrm{CO}_{2}$ (RPMI medium) and $5 \% \mathrm{CO}_{2}$ (M199, DMEM medium), respectively.

Preparation of low molecular weight DNA. Low-molecularweight (LMW) DNA was prepared by digestion with PlasmidSafe $^{\text {TM }}$ ATP-dependent DNase (Biozym Diagnostik, Hess. Oldendorf, Germany). At slightly alkine $\mathrm{pH}$-value this enzyme hydrolyzes predominantly linear double-stranded DNA and with lower efficiency, linear and closed-circular singlestranded DNAs into deoxynucleotides. However, it does not hydrolyze closed circular supercoiled nor nicked circular double-stranded DNAs. We digested $30 \mu \mathrm{g}$ of each DNA sample with 90 units Plasmid-Safe ${ }^{\mathrm{TM}}$ DNase according to the instructions of the supplier (Biozym Diagnostik).

Neutral-neutral two-dimensional (2D) gel electrophoresis. DNA was separated on neutral-neutral 2D gels according to the procedure described by Brewer and Fangman (13) with slight modifications. The first dimension was run in $0.4 \%$ agarose (without EtBr) at low voltage $(1 \mathrm{~V} / \mathrm{cm})$ in TAE buffer for $22 \mathrm{~h}$. Then the gel was rinsed in 1X TAE containing $0.3 \mu \mathrm{g} /$ $\mathrm{ml}$ EtBr. The lane of choice was cut out and placed horizontally in a clean gel support. A new gel with the cut out lane at the top was poured with $0.8 \%$ agarose containing $0.3 \mu \mathrm{g} / \mathrm{ml} \mathrm{EtBr}$. Rotated by 90 degrees to the direction of the first electrophoresis run, the second dimension was run in $1 \mathrm{X}$ TAE at $5 \mathrm{~V} / \mathrm{cm}$ for $6 \mathrm{~h}$.

Southern blotting. Southern blot analyses were carried out using Hybond- $\mathrm{N}^{+}$nylon membrane (Amersham Biosciences, Freiburg, Germany) according to standard techniques. Briefly, the gels were placed in denaturing buffer $(0.5 \mathrm{M}$ $\mathrm{NaOH}+1.5 \mathrm{M} \mathrm{NaCl}$ ) for $30 \mathrm{~min}$, then rinsed with destilled water and transferred to neutralizing buffer $(0.5 \mathrm{M}$ Tris- $\mathrm{HCl}$, $\mathrm{pH} 7.0+1.5 \mathrm{M} \mathrm{NaCl}$ ) for $30 \mathrm{~min}$. The gels were soaked in 20X SSC transfer buffer ( $3 \mathrm{M} \mathrm{NaCl}+0.3 \mathrm{M} \mathrm{Na}$ citrate) for 30 min. Blotting was done with a rapid downward transfer system Turboblotter ${ }^{\mathrm{TM}}$ (Schleicher \& Schüll, Dassel, Germany) for $5 \mathrm{~h}$. The transfer membrane was washed in 2X SSC for $5 \mathrm{~min}$, dried and baked at $80^{\circ} \mathrm{C}$ for $1 \mathrm{~h}$. 
Table II. Primer sequences.

\begin{tabular}{lccc}
\hline Primer name & PCR product size & Primer sequence (forward) & Primer sequence (reverse) \\
\hline Alu I & 200 bp fragment & 5'-GCCTGTAATCCCAGCACTTT-3' & 5'-GATCTCGGCTCACTGCAAC-3' \\
Alu II & 127 bp fragment & 5'-GACCATCCCGGCTAAAACG-3' & 5'-CGGGTTCACGCCATTCTC-3' \\
L1 I & 437 bp fragment & 5'-CTCCGGTCTACAGCTCCCAGCG-3' & 5'-ACCTAAGCAAGCCTGGGCAATG-3' \\
L1 II & 110 bp fragment & 5'-AGGTTGACAGCAGACTTATC-3' & 5'-GCTGGATATAGGATTCTAGG-3' \\
\hline
\end{tabular}

Hybridization with radiolabeled probes. Human Cot-1 DNA (Roche, Mannheim, Germany) served as a probe for the detection of total repetitive DNA molecules.

Human Alu-DNA probes were prepared by PCR generating either a 200 bp, named Alu I (14) or a 127 bp fragment, named Alu II (Acc.-No. EF372312; nucleotides 152-278). Human L1-DNA probes were prepared by PCR generating a 437 bp fragment, named L1 I (Acc.-No. AF149422; nucleotides 34-470), and a second human L1-DNA probe was prepared by PCR generating a 110 bp fragment, named L1 II (Acc.-No. NM_019072.3; nucleotides 2673-2782). All used primer sequences are listed in Table II. PCR was performed in a volume of $50 \mu \mathrm{l}$ containing $50 \mathrm{ng}$ of normal human genomic DNA, $20 \mathrm{pmol}$ of each primer, $200 \mu \mathrm{M}$ of each dNTP, 1X PCR buffer, $2.5 \mathrm{mM} \mathrm{MgCl}$, and 2.5 $\mathrm{U}$ of HotStarTaq DNA Polymerase (Qiagen, Hilden, Germany). The cycle regime consisted of an initial denaturation step at $95^{\circ} \mathrm{C}$ for $15 \mathrm{~min}$, followed by 35 cycles of $30 \mathrm{sec}$ at $95^{\circ} \mathrm{C}$, $30 \mathrm{sec}$ at the appropriate primer annealing temperature, $90 \mathrm{sec}$ at $72^{\circ} \mathrm{C}$ as well as a final extension step of 8 min at $72^{\circ} \mathrm{C}$ using a T3 Thermocycler (Biometra, Göttingen, Germany). The PCR products were separated on a $1.5 \%$ agarose gel and stained with $\mathrm{EtBr}$, the corresponding bands were cut out, and the DNA was isolated using the QIAquick gel extraction Kit (50) (Qiagen). All probes were labeled with Redivue $\left[\alpha^{-32} \mathrm{P}\right]-\mathrm{dCTP}$ (Amersham) using the Redprime II Random Prime Labeling System (Amersham) according to the manufacturer's instructions. Prehybridization of the membranes was done using the ExpressHyb ${ }^{\mathrm{TM}}$ hybridization solution (BD Biosciences Clontech, Heidelberg, Germany) at $65^{\circ} \mathrm{C}$ for $1 \mathrm{~h}$, and hybridization was carried out at $60^{\circ} \mathrm{C}$ (Cot-1 DNA) and $37^{\circ} \mathrm{C}$ (Alu-DNA, L1-DNA), respectively, for $1 \mathrm{~h}$.

As human telomeric DNA probe we used the sequence 5'TTAGGGTTAGGGTTAGGGTTAGGG-3' (TIB MOLBIOL, Berlin, Germany). The DNA was labeled with Redivue $\left[\alpha^{-32} \mathrm{P}\right]-$ dCTP (Amersham) using the terminal deoxynucleotidyl transferase and the terminal transferase buffer (Amersham) according to the manufacturer's instructions. The membranes were prehybridized at $65^{\circ} \mathrm{C}$ for $1 \mathrm{~h}$, followed by hybridization at $37^{\circ} \mathrm{C}$ for $1 \mathrm{~h}$. All radiolabeled membranes were exposed to a Fujifilm Imaging plate BAS-MS 2325 for $2 \mathrm{~h}$.

Densitometric analysis. Images for computer quantification were generated by the Phosphorimager Fuji FLA 3000 using the AIDA 2.11 software. The signal intensity obtained from the arc of linear DNA plus the arc of spcDNA is referred to as signal intensity of the total DNA. We calculated the percentage of the signal intensity of the spcDNA arc in relation to the signal intensity of the total arc.
Isolation of $s p c D N A$. The corresponding region was cut out from the agarose gel and the exact extraction of the spcDNA arc was subsequently confirmed by Southern blotting and hybridization. SpcDNA isolation was performed using the QIAquick gel extraction Kit (50) (Qiagen) as described above.

Cloning and sequencing of spcDNA. A phenol/chloroform purified spcDNA preparation containing 20-25 ng DNA was digested with $\mathrm{Bgl} \mathrm{II}$ and after inactivation at $80^{\circ} \mathrm{C}$, the DNA was digested with Klenow fragment according to the instructions of the supplier (Fermentas, St. Leon-Rot, Germany). Ligation was carried out either in the pJET1-Blunt vector (Fermentas) at room temperature for 5-30 $\mathrm{min}$, or in the pCR-Blunt vector (Invitrogen, Karlsruhe, Germany) at $16^{\circ} \mathrm{C}$ for $1 \mathrm{~h}$, using a T4 ligase under the conditions recommended by the supplier (GeneJET PCR Cloning Kit, Fermentas, and Zero Blunt PCR Cloning Kit, Invitrogen, respectively). The ligated DNA preparation was used to transform competent cells of Escherichia coli (TOP10). Transformed cells were selected for ampicillin and kanamycin resistance, respectively, and screened with X-gal and IPTG (Fermentas) for white colonies.

DNA of relevant clones was isolated with QIAprep Miniprep-Kit (Qiagen), purified and finally sequenced using BigDye Terminator v1.1 Cycle Sequencing Kit (Applied Biosystems, Darmstadt, Germany) on an ABI 370 automatic sequencer (Applied Biosystems).

\section{Results}

Identification of spcDNA. With the help of the above described electrophoretic separation technique two arcs were constantly detected: one very prominent arc represents the linear double-stranded DNA molecules, whereas the second arc was that of the relaxed spcDNA circles. Fig. 1 shows examples of Southern hybridization results obtained from the analyses of LMW-DNA of a healthy proband (Fig. 1A), and a cell line from a tumor tissue (Fig. 1B). We were able to detect spcDNA in the LMW-DNA of all 16 healthy probands as well as in the LMW-DNA of all 18 cell lines (Fig. 2). To get a quantitative value of the amount of spcDNA both arcs (linear and spcDNA) were quantified (using a Phosphoimager) and the amount of the spcDNA arc was expressed as a fraction of the sum of the intensities of the two arcs. It is evident that normal lymphocytes contain very low amounts of spcDNA and that the variability between the 16 individuals is small (ranging from 2.8 to $8.9 \%$ with an average of $5.4 \%$, Fig. 2). This is in contrast to the tumor cell lines which contain much 

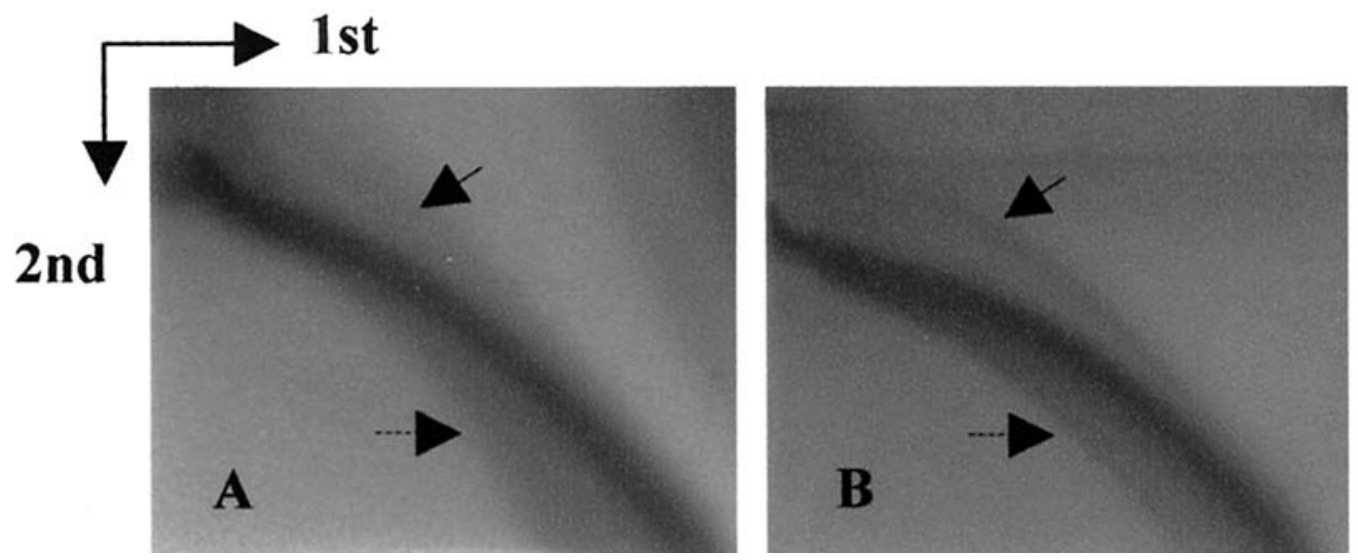

Figure 1. spcDNA of a healthy proband and a malignant cell line. LMW DNA of a healthy proband (A) and the osteogenic sarcoma cell line U-2 OS (B) were separated on a 2D gel. The gels were blotted and hybridized to a Cot-1 DNA probe. The very prominent arcs represent the linear double-stranded DNA molecules. The upper arcs represent relaxed circular molecules which were detected in all samples (solid arrows). The arcs below the linear DNA were identified as linear single-stranded DNA which arises by the preparation and is not cell specific (broken arrows).

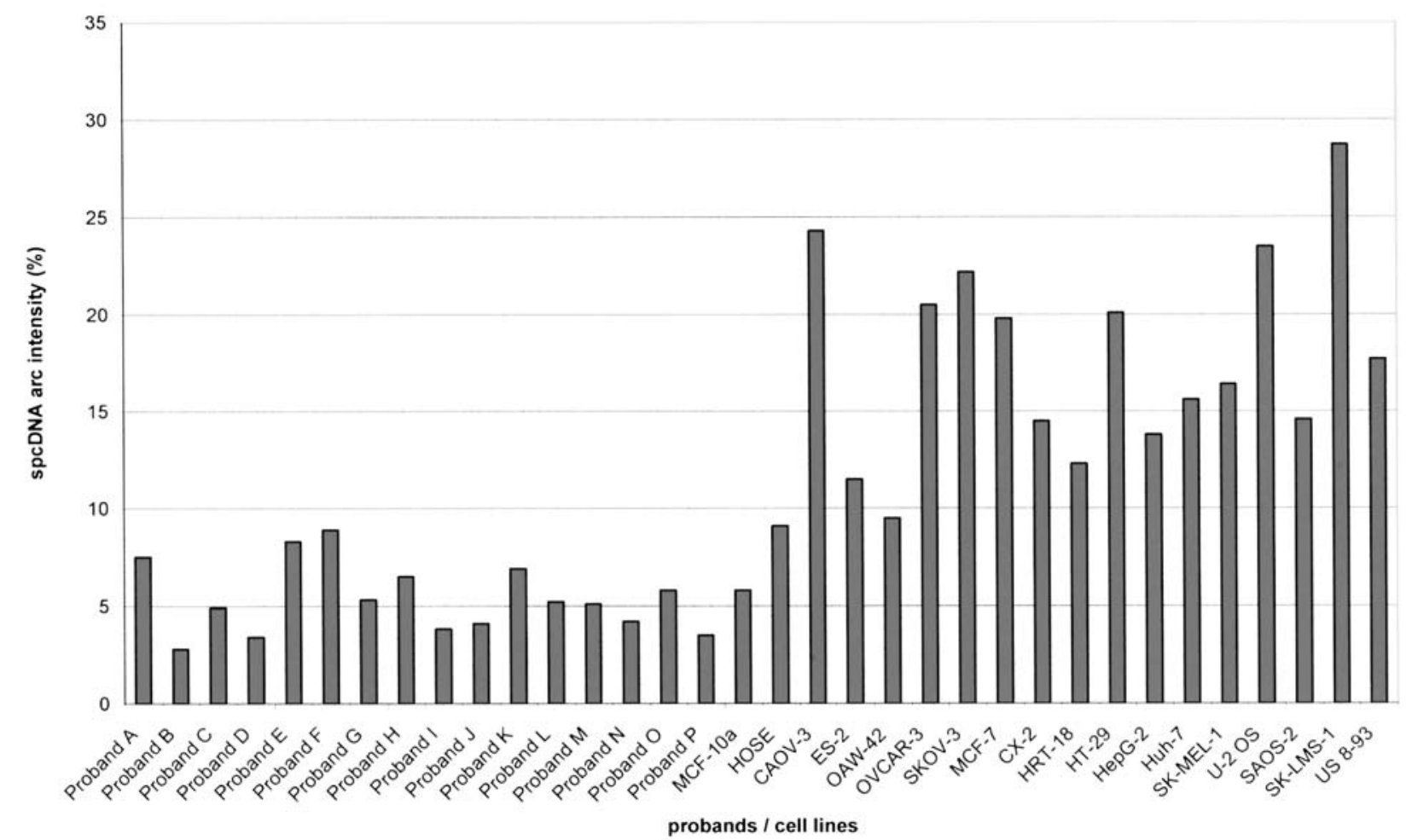

Figure 2. Distribution of spcDNA arc intensities in LMW DNA of lymphocytes of 16 healthy probands, two cell lines of normal tissues, and 16 cancer cell lines.

more spcDNA (on average 17.8\%) with a remarkable degree of variability (ranging from 9.5 up to $28.7 \%$ ). In between there are the two cell lines of normal human tissue (MCF10a from normal breast epithelium and HOSE from normal ovarian surface epithelium) with 5.8 and $9.1 \%$, respectively.

Detection of Alu, L1 and telomeric elements within the spcDNA. Southern blots were carried out with radiolabeled Alu, L1 and telomeric probes, respectively. We applied two non-overlapping Alu and L1 probes at a time to also detect fragmented Alu or L1 elements. The results are given in Table III. Fig. 3 shows examples of the Southern hybridi- zation results using DNA probes for Cot-1 (Fig. 3A), Alu (Fig. 3B), L1 (Fig. 3C), and telomeric sequences (Fig. 3D) on identical membranes of the ovarian adenocarcinoma cell line SKOV-3.

Except for the cell lines OAW-42, HepG-2, Huh-7, and SAOS-2 we found Alu sequences in the spcDNA of all the malignant tumor cell lines using the $200 \mathrm{bp}$ Alu fragment (Alu I) and/or the $127 \mathrm{bp}$ Alu fragment (Alu II) as a probe (Table III). Of the lymphocyte spcDNA of the 16 healthy probands only three were very weakly positive for Alu I and/or Alu II (Table III). In addition to their predominant absence in normal lymphocytes, Alu sequences were not 
Table III. Southern hybridization results with the probes for Alu, L1 and telomeric elements.

\begin{tabular}{|c|c|c|c|c|c|c|}
\hline \multirow[b]{2}{*}{ Case/cell line } & \multirow[b]{2}{*}{ Tissue origin } & \multicolumn{5}{|c|}{ Hybridization signal using sequences of } \\
\hline & & Alu I & Alu II & L1 I & L1 II & Tel \\
\hline Proband A & Human lymphocytes & & $(+)$ & & $(+)$ & \\
\hline Proband B & Human lymphocytes & & & & & \\
\hline Proband C & Human lymphocytes & & & & & \\
\hline Proband D & Human lymphocytes & & & & & \\
\hline Proband E & Human lymphocytes & $(+)$ & $(+)$ & $(+)$ & $(+)$ & \\
\hline Proband F & Human lymphocytes & $(+)$ & $(+)$ & $(+)$ & $(+)$ & \\
\hline Proband G & Human lymphocytes & & & & & \\
\hline Proband $\mathrm{H}$ & Human lymphocytes & & & & & \\
\hline Proband I & Human lymphocytes & & & & & \\
\hline Proband J & Human lymphocytes & & & & & \\
\hline Proband K & Human lymphocytes & & & & & \\
\hline Proband L & Human lymphocytes & & & & & \\
\hline Proband M & Human lymphocytes & & & & & \\
\hline Proband N & Human lymphocytes & & & & & \\
\hline Proband $\mathrm{O}$ & Human lymphocytes & & & & & \\
\hline Proband P & Human lymphocytes & & & & & \\
\hline HOSE & Ovarian epithelium & & & & + & \\
\hline CAOV-3 & Ovarian adenocarcinoma & + & + & + & + & \\
\hline ES-2 & Ovarian carcinoma & & + & + & + & \\
\hline OAW-42 & Ovarian adenocarcinoma & & & & + & \\
\hline OVCAR-3 & Ovarian adenocarcinoma & + & + & + & + & \\
\hline SKOV-3 & Ovarian adenocarcinoma & + & + & + & + & + \\
\hline MCF-10a & Breast epithelium (ductal) & & & & & \\
\hline MCF-7 & Breast adenocarcinoma & + & + & + & + & \\
\hline $\mathrm{CX}-2$ & Colon adenocarcinoma & + & + & + & + & \\
\hline HRT-18 & Colon adenocarcinoma & + & + & + & + & \\
\hline НТ-29 & Colon adenocarcinoma & & + & & + & \\
\hline HepG-2 & Hepatocellular carcinoma & & & & & \\
\hline Huh-7 & Hepatocellular carcinoma & & & + & + & \\
\hline SK-MEL-1 & Malignant melanoma & + & + & + & + & \\
\hline $\mathrm{U}-2$ OS & Osteogenic sarcoma & + & + & + & + & + \\
\hline SAOS-2 & Osteogenic sarcoma & & & + & + & + \\
\hline SK-LMS-1 & Leiomyosarcoma & + & + & + & + & + \\
\hline US 8-93 & Undifferentiated sarcoma & + & + & + & + & + \\
\hline
\end{tabular}

The cases with detectable Alu, L1 or telomeric sequences are marked with + . In the spcDNA of three probands we could detect a very weak positive signal of Alu and L1 sequences, marked with (+).

found in the cell lines from normal epithelial cells either (Table III).

With the help of the probes of a 437 bp L1 fragment (L1 I) and/or a $110 \mathrm{bp} \mathrm{L1}$ fragment (L1 II) all tumor cell lines but HepG-2, as well as the ovarian epithelium cell line HOSE revealed L1 sequences in their spcDNA (Table III). The same three cases of lymphocyte spcDNA that were positive for Alu-sequences showed very weak signals for the L1 probes I and/or II as well (Table III).
Telomeric sequences were not detected in normal lymphocytes or in the two normal tissue cell lines (MCF-10a and HOSE). However, six out of sixteen studied cancer cell lines contained telomeric sequences (Table III).

Cloning and sequencing of spcDNA. To answer the question after the presence of sequences other than those identified by Southern hybridization we isolated and cloned spcDNA, and sequenced 205 of the corresponding clones. The sequencing 

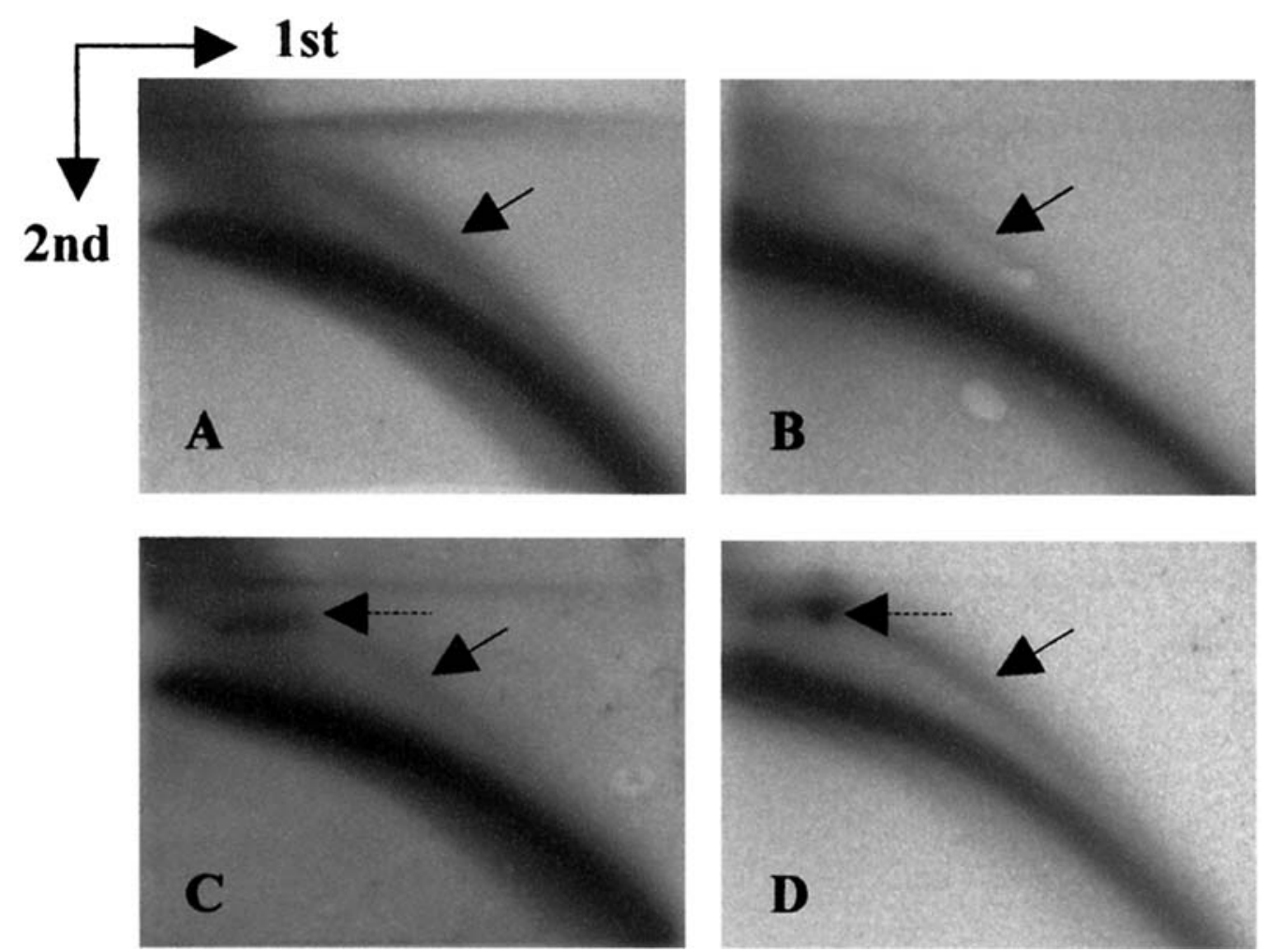

Figure 3. Southern hybridization results using probes of Cot-1, Alu, L1 and telomeric sequences. 2D gel electrophoresis, blotting of the gels and Southern hybridization of the membranes with LMW DNA from the ovarian adenocarcinoma cell line SKOV-3 using the Cot-1 probe (A), Alu probe (B), L1 probe (C) and telomeric probe (D). The solid arrows show the spcDNA. The broken arrows mark a region where the mitochondrial DNA is located in a $2 \mathrm{D}$ gel. The typical pattern of mitochondrial DNA can be easily distinguished from the linear DNA and from the arcs of the circular smaller molecules. It consists of a linear band and a non-linear smear representing the large circular structures (data not shown).

results revealed chromosomal sequences of almost all chromosomes, mostly from non-coding regions, but also a strong homology to two hypothetical proteins (hypothetical protein LOC745 on chromosome 11, LOC284434 on chromosome 19). One clone contained Alu sequences of the Alu-FLA, the Alu-J and the Alu-Sx elements.

\section{Discussion}

The neutral-neutral two-dimensional (2D) gel electrophoresis is a very useful tool for the identification and characterization of circular DNA molecules. By this technique, DNA molecules are separated both according to their size and their structure. Therefore, a population of molecules that are of the same structure, but of heterogeneous molecular mass, generates a continuous arc $(1,15)$. The structural identity of the DNA in each arc can be determined by electron microscopy $(15)$ and verified by molecular techniques $(6,7,15,16)$.

With the help of this electrophoretic separation technique we analysed a series of normal and tumor cell lines as well as normal uncultured human lymphocytes from healthy donors for their spcDNA contents to study the differences and common features of spcDNA in these systems. Furthermore, we focussed our detailed analysis of spcDNA on the highly repetitive Alu (small interspersed repetitive) and LINE-1 (large interspersed repetitive, also L1) elements as well as telomeric sequences to characterize them in their occurrence and frequency.
SpcDNA has been detected at an elevated level in genomically instable systems in contrast to stable systems of normal tissues. Those analyses were performed with DNA of patients with genetic diseases, for example Fanconi's anemia, in comparison with DNA of normal skin fibroblasts $(1,3)$. Other reports have shown that the elevated amount of spcDNA was apparently connected with increased aging $(17,18)$ and with transformation of cells $(1,4,15)$. Until now there is only one report that directly compares the spcDNA of normal skin fibroblasts with those of a colon carcinoma tissue and of the tumor cell line HeLa. However, spcDNA was not generally detectable in skin fibroblasts of this investigation (1).

In our studies, LMW DNA of healthy probands showed in all cases the occurrence of spcDNA, if at low level. These findings support the assumption that formation of spcDNA represents a normal physiological process in higher organisms $(6,9,19)$. For a long time it was thought that the eukaryote genome was relatively constant as a consequence of the highly regulated and ordered transmission of genetic information from generation to generation (2). However, it is now well known that genomes of eukaryotes are much more dynamic. The formation of extrachromosomal elements such as spcDNA makes it possible to respond efficiently to selective environmental influences or altering requirements during the life cycle of an organism (1).

In accordance to the above mentioned findings, we detected considerably more spcDNA in our tumor cell lines 
(a $>3$-fold higher amount) than in lymphocytes of healthy probands, and with a remarkable degree of variability.

Although the spcDNA situation in the two normal cell lines seems to be comparable to that of the lymphocytes, the corresponding arc intensity of HOSE was found to be somewhat higher than that of MCF-10a, even comparable to the tumor cell line with the lowest degree of spcDNA (OAW-42). This suggests that the spcDNA amount may not be fixed, but may rather fluctuate, especially in the genomic instable tumor cell lines.

Cloning and Southern hybridization of spcDNA fragments or total spcDNA have shown that there is a widely variable presence of repetitive sequences including satellite DNA, short interspersed and long interspersed repeat families, retrovirus-like elements, transposable elements, low copy, and single copy chromosomal sequences (2). Detection of Alu and L1 elements has been reported in analyses of spcDNA of rodent genomes [Chinese hamster (20), mouse (21-25)] and monkey genomes (26-28). But also in the human genome these sequences were identified, for example in human fibroblasts (29), and even more so in the human HeLa tumor cell line (2,30-32).

Alu and LINE sequences can be found in all genomic regions (33). They are the only active retrotransposons within the human genome, and it is assumed that the activation of retrotransposons and tumorigenesis are strictly connected (34). With our observation that an elevated level of spcDNA is associated with an increased level of Alu and/or L1 sequences in the spcDNA, one may suppose an indication of a mode of increasing retrotranspositional activity. Thus, spcDNA may indeed be regarded as a pool of fragmented extrachromosomal elements especially including L1.

Furthermore, previous reports have shown the presence of circular telomeric structures in different organisms under both normal and pathological circumstances $(4,7,9,35-37)$. Regev et al (4) found telomeric sequences in the spcDNA of human cells, especially in human colon carcinoma tissue and human hepatoma cell line, but not in the spcDNA of human breast carcinoma tissue and HeLa tumor cell line. However, for the first time we could show that in contrast to some cancer cell lines, normal lymphocytes of healthy probands do not seem to contain tel-spc at all.

To maintain telomere length, in most cases the telomerase is activated, which adds de novo telomeric repeats onto chromosomal termini (38). But also telomere elongation through telomerase-independent mechanisms have been described $(36,39)$. Those alternative lengthening of telomeres (ALT), mediated through homologous recombination is often accompanied by a generation of extrachromosomal telomeric circles (t-circles) $(40,41,42)$ which can serve as a reservoir for telomeric-repeat maintenance (37).

Previous studies have shown that the ALT mechanism is used more frequently in tumors of mesenchymal origin than in those derived from the epithelium (41). All sarcoma cell lines investigated in this study carried tel-spcDNA. This finding could refer to the ALT mechanism of telomere lengthening as it has been described for cell line SAOS-2 $(43,44)$. The significance of telomeric circles remains unclear, but it is suggested that they are actively involved in telomere dynamics and in genomic instability $(4,36,40)$. Tel-spcDNA may have a potential role as mutator in genomic instability (4). However, findings of others and our results suggest that not only telomere, but the whole spcDNA including retrotranspositional elements may play a role for chromosomal rearrangements and genomic instability that occur both in normal physiological and pathological processes.

It has already been shown that cloned spcDNA fragments can share homologies with chromosomal DNA sequences, containing both repetitive sequences and unique chromosomal sequences (2). Our cloning and sequencing results of spcDNA revealed in most cases chromosomal sequences of almost all chromosomes, but we could not observe an increased frequency of any single region. One clone contained different elements of the repetitive Alu sequence family, i.e., Alu-FLA, Alu-J and Alu-Sx elements.

To date, predominantly repetitive sequences were reported in the formation of spcDNA (45). Detection and characterizations of single or low copy chromosomal sequences were rare. Only one report exists on spcDNA comprising a partial region from the $P I P$ gene, a gene which is expressed in primary breast carcinoma (5). The function of the two hypothetical proteins LOC745 and LOC284434 to which we detected a strong homology is unknown yet. LOC284434 has also been named as NACHT and WD repeat domain containing 1 protein. The group of Lengauer reported a relation between chromosome instability and mutations in another WD repeat-containing protein, $\mathrm{CDC} 4$, in colorectal cancer (46). Further investigations, especially of the non-repetitive sequences in the spcDNA, could unravel the significance of those circular DNA structures as a reservoir and/or the starting point for gene amplification and chromosome instability in cancer development and progression.

In summary, spcDNA occurs both in lymphocytes of healthy probands and in tumor cell lines. However, it is the quantity and, to some extent, the sequence composition that differs. So, the amount of spcDNA in tumor cell lines can be more than 3-fold higher than in lymphocytes of healthy probands. Since we could furthermore detect repetitive sequences including Alu, L1 and telomeric elements in spcDNA by Southern hybridization we suggest that an increased amount of spcDNA can be associated with genomic instability occurring during tumorigenesis.

\section{Acknowledgements}

We thank Mrs. Ilona Wiederhold for her excellent technical assistance. H.T. was supported by the Dt. Krebshilfe (107590).

\section{References}

1. Cohen S, Regev A and Lavi S: Small polydispersed circular DNA (spcDNA) in human cells: association with genomic instability. Oncogene 14: 977-985, 1997.

2. Gaubatz JW: Extrachromosomal circular DNAs and genomic sequence plasticity in eukaryotic cells. Mutat Res 237: 271-292, 1990.

3. Motejlek K, Schindler D, Assum G and Krone W: Increased amount and contour length distribution of small polydisperse circular DNA (spcDNA) in Fanconi anemia. Mutat Res 293: 205-214, 1993.

4. Regev A, Cohen S, Cohen E, Bar-Am I and Lavi S: Telomeric repeats on small polydisperse circular DNA (spcDNA) and genomic instability. Oncogene 17: 3455-3461, 1998. 
5. Autiero M, Camarca A, Ciullo M, Debily MA, El Marhomy S, Pasquinelli R, Capasso I, D'Aiuto G, Anzisi AM, PiatierTonneau D and Guardiola J: Intragenic amplification and formation of extrachromosomal small circular DNA molecules from the PIP gene on chromosome 7 in primary breast carcinomas. Int J Cancer 99: 370-377, 2002.

6. Cohen S, Menut S and Mechali M: Regulated formation of extrachromosomal circular DNA molecules during development in Xenopus laevis. Mol Cell Biol 19: 6682-6689, 1999.

7. Cohen S and Mechali M: Formation of extrachromosomal circles from telomeric DNA in Xenopus laevis. EMBO Rep 3: 1168-1174, 2002.

8. Cohen Z, Bacharach E and Lavi S: Mouse major satellite DNA is prone to eccDNA formation via DNA ligase IV-dependent pathway. Oncogene 25: 4515-4524, 2006.

9. Cohen S, Houben A and Segal D: Extrachromosomal circular DNA derived from tandemly repeated genomic sequences in plants. Plant J 53: 1027-1034, 2008.

10. Soule HD, Maloney TM, Wolman SR, Peterson WD Jr, Brenz R, McGrath CM, Russo J, Pauley RJ, Jones RF and Brooks SC: Isolation and characterization of a spontaneously immortalized human breast epithelial cell line, MCF- $10^{1}$. Cancer Res 50: 6075-6086, 1990.

11. Tsao S-W, Mok SC, Fey EG, Fletcher JA, Wan TSK, Chew E-C, Muto MG, Knapp RC and Berkowitz RS: Characterization of human ovarian surface epithelial cells immortalized by human papilloma viral oncogenes (HPV-E6E7 ORFs). Exper Cell Res 218: 499-507, 1995

12. Sambrook J, Fritsch EF and Maniatis T (eds): Molecular cloning: a laboratory manual. 2nd edition. Cold Spring Harbor Laboratory, Cold Spring Harbor, New York, 1989.

13. Brewer BJ and Fangman WL: The localization of replication origins on ARS plasmids in S. cerevisiae. Cell 51: 463-471, 1987.

14. Hagan CR, Sheffield RF and Rudim CM: Human Alu element retrotransposition induced by genotoxic stress. Nat Genet 35: 219-220, 2003

15. Cohen S and Lavi S: Induction of circles of heterogenous sizes in carcinogen-treated cells: two-dimensional gel analysis of circular DNA molecules. Mol Cell Biol 16: 2002-2014, 1996.

16. Cohen S and Mechali M: A novel cell-free system reveals a mechanism of circular DNA formation from tandem repeats. Nucleic Acid Res 29: 2542-2548, 2001.

17. Kunisada T, Yamagishi H, Ogita Z, Kirakawa T and Mitsui Y: Appearance of extrachromosomal circular DNAs during in vivo and in vitro ageing of mammalian cells. Mech Ageing Dev 29: 89-99, 1985.

18. Yamagishi H, Kunisada $\mathrm{T}$ and Takeda T: Amplification of extrachromosomal small circular DNAs in a murine model of accelerated senescence. A brief note. Mech Ageing Dev 29: 101-103, 1985

19. Cohen S, Yacobi K and Segal D: Extrachromosomal circular DNA of tandemly repeated genomic sequences in Drosophila. Genome Res 13: 1133-1145, 2003.

20. Miles C and Meuth M: Hamster line and Alu-equivalent sequences are present in the small polydispersed circular DNA population of CHO cells. Nucleic Acids Res 17: 5846, 1989.

21. Fujimoto S, Tsuda T, Toda M and Yamagishi H: Transposonlike sequences in extrachromosomal circular DNA from mouse thymocytes. Proc Natl Acad Sci USA 82: 2072-2076, 1985.

22. Sunnerhagen P, Sjöberg RM, Karlsson AL, Lundh L and Bjursell G: Molecular cloning and characterization of small polydisperse circular DNA from mouse 3 T6 cells. Nucleic Acids Res 14: 7823-7838, 1986.

23. Sunnerhagen P, Sjöberg RM and Bjursell G: Increase of extrachromosomal circular DNA in mouse 3T6 cells on perturbation of DNA synthesis: implications for gene amplification. Somat Cell Mol Genet 15: 61-70, 1989.

24. Flores SC, Sunnerhagen P, Moore TK and Gaubatz JW: Characterization of repetitive sequence families in mouse heart small polydisperse circular DNAs: age-related studies. Nucleic Acids Res 16: 3889-3906, 1988.

25. Gaubatz JW and Flores SC: Tissue-specific and age-related variations in repetitive sequences of mouse extrachromosomal circular DNAs. Mutat Res 237: 29-36, 1990.
26. Bertelsen AH, Humayun MZ, Karfopoulos SG and Rush MG: Molecular characterization of small polydisperse circular deoxyribonucleic acid from an African green monkey cell line. Biochemistry 21: 2076-2085, 1982.

27. Krolewski JJ, Schindler CW and Rush MG: Structure of extrachromosomal circular DNAs containing both the Alu family of dispersed repetitive sequences and other regions of chromosomal DNA. J Mol Biol 174: 41-54, 1984.

28. Schindler CW and Rush MG: Discrete size classes of monkey extrachromosomal circular DNA containing the L1 family of long interspersed nucleotide sequences are produced by a general non-sequence specific mechanism. Nucleic Acids Res 13: 8247-8258, 1985 .

29. Riabowol K, Shmookler Reis RJ and Goldstein S: Interspersed repetitive and tandemly repetitive sequences are differentially represented in extrachromosomal covalently closed circular DNA of human diploid fibroblasts. Nucleic Acids Res 13: 5563-5584, 1985.

30. Kunisada T and Yamagishi H: Sequence repetition and genomic distribution of small polydisperse circular DNA purified from HeLa cells. Gene 31: 213-223, 1984.

31. Kunisada T and Yamagishi H: Sequence organization of repetitive sequences enriched in small polydisperse circular DNAs from HeLa cells. J Mol Biol 198: 557-565, 1987.

32. Jones RS and Potter SS: Characterization of cloned human alphoid satellite with an unusual monomeric construction: evidence for enrichment in HeLa small polydisperse circular DNA. Nucleic Acids Res 13: 1027-1042, 1985.

33. Kazazian HH Jr and Goodier JL: LINE drive. Retrotransposition and genome instability. Cell 110: 277-280, 2002.

34. Hauptmann S and Schmitt WD: Transposable elements - is there a link between evolution and cancer? Med Hypotheses 66: 580-591, 2006.

35. Horowitz $\mathrm{H}$ and Haber JE: Identification of autonomously replicating circular subtelomeric Y' elements in Saccharomyces cerevisiae. Mol Cell Biol 5: 2369-2380, 1985.

36. Tomaska L, McEachern MJ and Nosek J: Alternatives to telomerase: keeping linear chromosomes via telomeric circles. FEBS Lett 567: 142-146, 2004.

37. Larrivee $M$ and Wellinger RJ: Telomerase- and cappingindependent yeast survivors with alternate telomere status. Nat Cell Biol 8: 741-747, 2006.

38. McEachern MJ, Iyer S, Fulton TB and Blackburn EH: Telomere fusions caused by mutating the terminal region of telomeric DNA. Proc Natl Acad Sci USA 97: 11409-11414, 2000.

39. Dunham MA, Neumann AA, Fasching CL and Reddel RR: Telomere maintenance by recombination in human cells. Nat Genet 26: 447-450, 2000.

40. Nosek J, Rycovska A, Makhov AM, Griffith JD and Tomaska L: Amplification of telomeric arrays via rolling-circle mechanism. J Biol Chem 280: 10840-10845, 2005.

41. Johnson JE and Broccoli D: Telomere maintenance in sarcomas. Curr Opin Oncol 19: 377-382, 2007.

42. Compton SA, Choi JH, Cesare AJ, Ozgür S and Griffith JD: $\mathrm{Xrcc} 3$ and Nbs1 are required for the production of extrachromosomal telomeric circles in human alternative lengthening of telomere cells. Cancer Res 67: 1513-1519, 2007.

43. Gan Y, Mo Y, Johnston J, Lu J, Wientjes MG and Au JL-S: Telomere maintenance in telomerase-positive human ovarian SKOV-3 cells cannot be retarded by complete inhibition of telomerase. FEBS Lett 527: 10-14, 2002.

44. Tsai H-J, Huang W-H, Li T-K, Tsai Y-L, Wu K-J, Tseng S-F and Teng S-C: Involvement of topoisomerase III in telomeretelomere recombination. J Biol Chem 281: 13717-13723, 2006.

45. Kuttler F and Mai S: Formation of non-random extrachromosomal elements during development, differentiation and oncogenesis. Semin Cancer Biol 17: 56-64, 2007.

46. Rajagopalan H, Jallepalli PV, Rago C, Velculescu VE, Kinzler KW, Vogelstein B and Lengauer C: Inactivation of hCDC4 can cause chromosomal instability. Nature 428: 77-81, 2004. 